\title{
Primary Aldosteronism and Obstructive Sleep Apnea: Casual Association or Pathophysiological Link?
}

Authors

Alessio Pecori, Fabrizio Buffolo, Jacopo Pieroni, Vittorio Forestiero, Elisa Sconfienza, Franco Veglio, Paolo Mulatero@, Silvia Monticone

\author{
Affiliation \\ Division of Internal Medicine 4 and Hypertension Unit, \\ Department of Medical Sciences, University of Torino, \\ Torino, Italy
}

Key words

aldosterone, sleep disorders, obesity, metabolic syndrome, apnea-hypopnea index, leptin

received $\quad 18.12 .2019$

accepted 26.02.2020

\author{
Bibliography \\ DOI https://doi.org/10.1055/a-1133-7255 \\ Published online: 27.3.2020 \\ Horm Metab Res 2020; 52: 366-372 \\ (c) Georg Thieme Verlag KG Stuttgart · New York \\ ISSN 0018-5043 \\ Correspondence \\ Paolo Mulatero MD \\ Division of Internal Medicine 4 and Hypertension Unit \\ Department of Medical Sciences, University of Torino \\ Via Genova 3 \\ 10126 Torino \\ Italy \\ Tel.: + 390116336959 , Fax: + 390116336931 \\ paolo.mulatero@unito.it
}

\section{ABSTRACT}

The coexistence of aldosterone oversecretion and obstructive sleep apnea is frequently observed, especially in patients with resistant hypertension, obesity, and metabolic syndrome. Since aldosterone excess and sleep apnea are both independently associated with an increased risk of cardiovascular disease, to investigate whether their coexistence might be attributed to common predisposing conditions, such as metabolic disorders, or to an actual pathophysiological interconnection appears of great importance. Fluid overload and metabolic abnormalities relating to aldosterone oversecretion may be implicated in obstructive sleep apnea development. Nocturnal intermittent hypoxia may in turn exacerbate renin-angiotensin-aldosterone system activity, thus leading to hyperaldosteronism. Furthermore, fat tissue excess and adipocyte secretory products might predispose to both sleep apnea and aldosterone oversecretion in subjects with obesity. Consistent with these evidences, obstructive sleep apnea frequently affects patients with primary aldosteronism. Conversely, whether primary aldosteronism is more prevalent in individuals affected by obstructive sleep apnea compared to the general population remains controversial.

\section{Introduction}

Obstructive sleep apnea (OSA), aldosterone excess, and resistant hypertension $(\mathrm{RH})$ are common comorbidities, especially in patients affected by obesity [1]. The prevalence of OSA is high in patients affected by $\mathrm{RH}$ and even higher in individuals with hypertension refractory to medical therapy [2]. In patients with $\mathrm{RH}$, oversecretion of aldosterone is a frequent finding and primary aldosteronism (PA) has a significantly higher prevalence compared with the general hypertensive population [3]. Patients with $\mathrm{RH}$ are significantly more likely to experience adverse cardiovascular outcomes, such as stroke, myocardial infarction, heart failure, renal failure, and death, compared with subjects with nonresistant hypertension [4]. Studies performed in the last years suggest that $P A$ is frequent in patients with OSA [5, 6] and, on the basis of these evidences, the 2016 En- docrine Society Guideline recommends to screen for PA all patients with hypertension and OSA [7]. The impact of aldosterone excess and sleep apnea on the cardiovascular morbidity and mortality of patients with hypertension is of great importance, as well as the recognition of the interplay between these conditions. Previous studies hypothesized that a bidirectional relationship between aldosterone levels and OSA might be present in patients with $\mathrm{RH}$ and PA [8]. Nevertheless, whether the association between PA and OSA is accidental or pathophysiologically-based remains controversial.

\section{Epidemiology}

Primary aldosteronism

PA is mostly sporadic, due to unilateral aldosterone-producing adenoma (APA) or bilateral hyperaldosteronism (BHA), and less fre- 
quently (1-6\%) is a familiar condition [9]. Individuals with PA show an increased risk of stroke, coronary artery disease, heart failure, atrial fibrillation, left ventricular hypertrophy, kidney damage, diabetes, and metabolic syndrome compared to patients with essential hypertension $[10,11]$. The prevalence of PA rates between $5.9 \%$ in the general hypertensive population [12] and $11.2 \%$ in patients from referral centers [13], and progressively increases with hypertension severity [12].

Two prospective studies suggested a high prevalence of aldosterone excess in patients with sleep disordered breathing (SDB). Calhoun et al. showed that autonomous aldosterone secretion was more frequently observed in patients with $\mathrm{RH}$ at high risk of OSA (36\%) compared with low-risk patients (19\%), defined according to the Berlin Questionnaire results [5]. However, OSA diagnosis was not confirmed by polysomnography and patients with aldosterone oversecretion did not underwent confirmatory testing for PA diagnosis. Di Murro et al. reported a 34\% prevalence of PA in patients affected by hypertension and OSA [6]. Nonetheless, polysomnography was performed only in patients showing excessive daytime sleepiness, thus leading to diagnosis of OSA only in a selected subgroup of symptomatic individuals. Therefore, it cannot be excluded that PA prevalence among the overall symptomatic and asymptomatic OSA population would have been different.

The recent multicenter multi-ethnic cross-sectional HYPNOS study, reported an $8.9 \%$ prevalence of PA in a population of 203 patients with OSA (102 Caucasians and 101 Chinese) [14]. Even though control groups were not included in the study, the figures were not significantly different from the prevalence of PA reported in primary care settings and referral centers (5.9 and $11.2 \%$, respectively) $[12,13]$. Moreover, a sub-analysis performed to identify PA prevalence in patients in whom OSA was the only indication to proceed to PA screening, showed that only $1 / 63$ (1.5\%) patients displayed confirmed PA. These results provocatively challenge the current Endocrine Society Guideline recommendation to screen for PA all patients affected by OSA and arterial hypertension, irrespective of hypertension severity and the presence of other risk factors.

\section{Obstructive sleep apnea}

OSA is a chronic sleep disorder characterized by recurrent episodes of complete (apneas) or partial airflow cessation (hypopneas) due to obstruction of the upper respiratory tract, leading to intermittent hypoxia, hypercapnia, and sleep arousals [15]. OSA is an independent risk factor for hypertension [16-18] and cardiovascular disease $[19,20]$, and a predictor of cardiovascular outcomes, including sudden cardiac death [21]. OSA is frequently diagnosed in patients with various cardiovascular comorbidities, such as stroke, end-stage chronic kidney disease, ischemic cardiomyopathy, heart failure, atrial fibrillation, and hypertrophic cardiomyopathy [15]. The prevalence of OSA in the overall adult population varies from $9-38 \%$, ranging between $13-33 \%$ in men and $6-19 \%$ in women [22]. Advanced age is associated with an elevated prevalence of OSA, up to $90 \%$ for all stages in a cohort of elderly men [22, 23]. OSA prevalence is high in patients with obesity or metabolic syndrome, ranging from $50 \%$ to $60 \%$, and it is even higher in subjects with morbid obesity and diabetes [24]. The prevalence of OSA in patients with hypertension is reported to be $30-50 \%$ [25] and it has been assessed to be as high as $92 \%$ [26] for all stages and $70 \%$ for severe OSA [27] in patients with $\mathrm{RH}$. A recent study displayed an even higher prevalence of moderate OSA (95.2\%), severe OSA (64.3\%), and OSA syndrome (52.4\%) in patients with refractory hypertension [2].

Some studies showed that OSA is more frequent in patients with than in those without PA. In a retrospective cohort, OSA prevalence was significantly higher in subjects with autonomous aldosterone secretion (18\%) compared with patients without it (9\%) [28]. However, the lack of PA confirmatory testing and the identification of OSA patients using database records are the major limitations of this study. Indeed, the prevalence of OSA in both PA and non-PA hypertensive patients resulted even lower than the one observed in the general population [22]. In a prospective study, the reported OSA prevalence was 78.1 and $71.0 \%$ in patients affected by $\mathrm{RH}$ with and without PA, respectively, and OSA severity was shown to be higher in the former group [29]. In a recent study aimed at exploring the effects of PA treatment on OSA severity, a 79\% prevalence of OSA was showed in patients with PA [30]. Nevertheless, it should be noted that the study cohort was selected by the presence of at least one risk factor for sleep apnea, such as male gender, obesity, history of snoring or daytime sleepiness, therefore the assessed prevalence might represent an overestimation of the actual OSA prevalence in the overall PA population because of selection bias. In the HYPNOS study, among 207 patients with a confirmed diagnosis of PA, the prevalence of OSA was 67.6\% [14].

Given the high cardiovascular morbidity associated with both PA $[10,11]$ and OSA [16-21], it is possible that the coexistence of both diseases could act synergistically further increasing the associated cardiovascular risk and target organ damage. Data from HYPNOS study showed that patients with both PA and OSA have higher blood pressure levels than PA or OSA alone [14]. However, no studies directly investigated the combined effect of OSA and PA in terms of target organ damage and cardiovascular risk.

\section{Ethnic differences in primary aldosteronism and} obstructive sleep apnea prevalence

Whether ethnic differences might affect the risk of both OSA and PA onset has been a matter of debate. The prevalence of OSA in the general Caucasian population was estimated to be as high as $38 \%$ to even more than $90 \%$ in selected cohorts $[2,22,23]$ and it was observed to be $64.4 \%$ in patients with PA [14]. The prevalence of PA was found to be $5.9 \%$ in the general hypertensive population [12] and $11.8 \%$ in Caucasian patients with OSA [14].

The SDB risk in Asian patients was reported to be higher than in Caucasians [31]. OSA is more frequent in Chinese men than in women, although prevalence discrepancies lower along with age $[32,33]$. The elevated SDB risk in Asians may be correlated to anatomical factors, such as the higher frequency of craniofacial skeletal anomalies and the different body fat distribution compared to Caucasians [31]. Asian patients usually display a greater dietary salt intake than Caucasians, and this feature, along with an elevated salt-sensitivity of blood pressure, might contribute to hydrosaline retention and pharyngeal edema [34]. In the HYPNOS study, Chinese patients with PA had a $70 \%$ prevalence of OSA, which was not significantly different compared with Caucasians [14], and resulted to be similar to that reported in Chinese with essential hyper- 
tension [35]. Moreover, aldosterone positively correlated with apnea-hypopnea index (AHI) in Caucasians with PA, but not in Chinese patients [14]. Hence, it is reasonable to suppose that the pathophysiology of OSA in Asians might be related mostly to anthropomorphic predisposing features and high salt intake than to aldosterone-mediated mechanisms.

Current evidences suggest that SDB risk is greater among patients of African descent compared to Caucasians [31, 36, 37]. SDB predisposition might be correlated to obesity influence and presence of enlarged upper airway soft tissue in blacks, as well as frequent disadvantageous socio-environmental conditions and tobacco smoke [31]. Blacks with hypertension tend to have lower plasma renin activity than whites [38], possibly suggesting a greater frequency of aldosterone-dependent hypertension [3], though no differences in PA prevalence have been observed [3, 39]. Moreover, blacks tend to be more salt-sensitive and to display lower responsiveness to angiotensin-converting enzyme inhibitors and beta-blockers compared to whites [40]. These evidences possibly suggest that aldosterone excess might play a relevant role in the pathophysiology of OSA in African-Americans, as supported by a study reporting a higher prevalence of both OSA and PA compared with Caucasians, Hispanics, and Asians [28].

\section{The Pathophysiological link between aldosterone and OSA}

Obstructive sleep apnea effects on the renin-angiotensinaldosterone system

Several pathophysiological mechanisms relating OSA to hypertension have been proposed, including intermittent hypoxia, hypercapnia, intrathoracic negative pressure changes and nocturnal arousals. Blood pressure increase due to intermittent hypoxic events has been associated to sympathetic nervous system hyperactivity, renin-angiotensin-aldosterone system (RAAS) overstimulation, oxidative stress, endothelial dysfunction, endothelin overproduction and pro-inflammatory state [41]. OSA severity might correlate with aldosterone oversecretion, thus leading to water and sodium retention, and subsequent rise in blood pressure. Indeed, patients with OSA and $\mathrm{RH}$ with normal plasma renin activity, plasma aldosterone concentration and aldosterone-to-renin ratio displayed a reduced aldosterone response to saline load along with increasing severity of OSA [42].

Is aldosterone excess a result of RAAS hypoxia-induced activation in patients with OSA, or does it represent a contributing factor to OSA pathophysiology? To assess causality of OSA and aldosterone excess relationship, a number of clinical studies were performed and evaluated the impact of continuous positive airway pressure (CPAP) treatment on aldosterone levels in patients with OSA. The results of a meta-analysis of 5 studies indicated minimally yet significantly reduced aldosterone levels after at least one month of CPAP therapy. Nonetheless, a sub-analysis including only randomized controlled trials indicated no significant aldosterone changes in the CPAP-treated patients compared to controls [43]. However, the study performed by Møller et al., showing no significant decrease of aldosterone levels after CPAP therapy, might be limited by the lack of an appropriate BMI-matching of enrolled subjects, thus leading to biased results due to obesity influence on
CPAP effects [44]. Of interest, all included studies were performed in Europe and enrolled patients were predominantly male, thus ethnicity and gender influence on aldosterone response to CPAP treatment could not be assessed.

A recent study showed that also short-term CPAP treatment might be able to lower plasma aldosterone and renin levels in moderate-severe OSA patients with type 2 diabetes [45]. Conversely, a recent randomized controlled trial reported that only optimal CPAP treatment - at least $4 \mathrm{~h}$ per night during the entire 6-month study period - significantly decreased urinary aldosterone excretion in $\mathrm{RH}$ patients with moderate-severe OSA compared to controls [46]. Another study displayed that CPAP therapy was able to significantly decrease aldosterone levels and blood pressure in OSA patients with day-and-night sustained hypertension compared to controls, but not in those with isolated nocturnal hypertension [47]. Lastly, aldosterone levels were not significantly decreased by angiotensin receptor-blocker treatment in a cohort of subjects with hypertension and OSA compared to those without OSA, while add-on CPAP therapy in the former group tended to lower aldosterone excess and sympathetic activity. These results support the hypothesis of a subclinical form of hyperaldosteronism in patients with hypertension and OSA [48].

\section{Overnight rostral fluid shift as a pathogenetic feature predisposing to obstructive sleep apnea}

Several evidences support the importance of volume overload and nocturnal rostral fluid shift in the pathogenesis of OSA. This latter phenomenon is due to redistribution of fluid, accumulated during daytime in the lower extremities because of gravity, from legs to neck upon lying down at night. Fluid displacement into neck structures promotes upper respiratory tract soft tissue edema, which in turn causes airway resistance increase and airflow obstruction [49].

Targeting fluid overload in hypervolemic patients might be effective to reduce $\mathrm{AHI}$ and upper airway collapsibility in subjects with OSA [49]. A 2-week course of intensified diuretic treatment was reported to be effective in improving OSA severity in patients with uncontrolled hypertension and moderate-severe OSA [50].

Aldosterone excess, along with increased dietary sodium intake, leads to fluid overload $[1,51]$. A meta-analysis showed that aldosterone and angiotensin II are significantly higher in OSA-affected patients with hypertension than in normotensive individuals [52]. Therefore, it has been hypothesized that aldosterone excess might be implicated in volume overload predisposing nocturnal rostral fluid shift in patients with OSA [51]. Conversely, in a large cross-sectional study plasma aldosterone levels and plasma renin activity were not significantly different in $\mathrm{RH}$ patients with no or mild OSA compared with patients with moderate-severe OSA [53].

\section{Aldosterone blockade and obstructive sleep apnea}

Several studies documented a positive correlation between aldosterone levels and OSA severity. Plasma and urinary aldosterone levels significantly correlated with $\mathrm{AHI}$ in subjects with $\mathrm{RH}$, but this correlation was not observed in normotensive individuals nor in patients with treatment-controlled hypertension of similar age, BMI and OSA severity $[46,54]$. Nevertheless, a study reported a positive correlation between aldosterone levels and $\mathrm{AHI}$ in patients 
affected by essential, but not secondary, RH [29]. Some studies reported that plasma and urinary aldosterone levels positively correlate with $\mathrm{AHI}$ in patients with $\mathrm{RH}$ and autonomous aldosterone production. Conversely, no significant correlation between aldosterone levels and $\mathrm{AHI}$ was observed in patients with normal aldosterone secretion, suggesting that the role of aldosterone in OSA pathogenesis and severity might be relevant only in subjects displaying a status of aldosterone excess $[55,56]$.

A number of clinical studies evaluated the effect of aldosterone blockade on airway obstruction. Two small observational studies reported a significant reduction of $\mathrm{AHI}$ after a 2-month treatment with spironolactone in patients with $\mathrm{RH}$ and moderate-severe OSA [57], and a 3-month treatment with eplerenone in patients with essential RH and OSA, respectively [58]. Likewise, a small randomized blank-controlled prospective trial showed a significant reduction of OSA severity, blood pressure and aldosterone levels in patients affected by RH and moderate-severe OSA after a 3-month spironolactone therapy on top of preexisting antihypertensive treatment compared to controls [59]. Furthermore, AHI and neck circumference were reduced by PA medical or surgical therapy in patients affected by both PA and OSA [30].

Aldosterone excess might concur to airway obstruction pathogenesis not only by fluid overload-mediated mechanisms, but also through direct impairment and deregulation of central ventilatory-control $[41,60]$. Nowadays, targeting aldosterone excess has not yet been defined as an effective treatment for patients with OSA. Notwithstanding, mineralocorticoid receptor-antagonist treatment should be considered in patients with OSA and RH [41].

Primary aldosteronism, obstructive sleep apnea and obesity

The coexistence of high aldosterone levels and OSA might also be attributed to the presence of common predisposing conditions, such as obesity and metabolic disorder [61]. OSA prevalence is elevated in individuals affected by obesity or metabolic syndrome, and it is even higher in subjects with diabetes and morbid obesity [24]. Furthermore, OSA is associated with insulin resistance independently of concomitant obesity [62] and abnormal glucose metabolism may be attributed to hypoxemia-induced sympathetic nervous system and RAAS activation [63]. Diabetic neuropathy may in turn affect central control of respiration promoting sleep apnea development, leading to a vicious cycle [64].

Obesity is common in patients with both OSA and RH [1]. Interestingly, aldosterone levels appear to be higher in patients with OSA and metabolic syndrome compared to those without metabolic alterations [65]. Several studies observed high aldosterone levels in obese subjects, especially in those with visceral obesity [66]. Both adolescents and women with obesity displayed significantly higher aldosterone levels than lean individuals, and weight loss led to significant aldosterone reduction $[67,68]$. A recent study demonstrated that urinary aldosterone levels were positively correlated with $\mathrm{BMI}$ in $\mathrm{RH}$ patients, especially in men and regardless of ethnicity [69]. Moreover, BMI was directly correlated to aldosterone levels in postmenopausal Chinese women with obesity and hypertension but not in premenopausal women, possibly because of the role of endogenous estrogens as a regulator of aldosterone secretion before menopause [70].
The pathophysiological pathways linking obesity to aldosterone excess still have to be fully elucidated. Adipose cells are able to produce angiotensinogen and angiotensin II; studies on animal models showed that angiotensinogen might be released into circulation by adipose tissue and its levels might correlate with systolic blood pressure [71]. Animal model studies suggested that high concentrations of fatty acids in portal venous blood of obese individuals might be metabolized by the liver to oxidized products, such as linoleic acid derivatives, which could stimulate aldosterone secretion [72]. Adipocyte secretory products, such as adipokines and adipocyte-derived hormones, may directly stimulate adrenocortical aldosterone secretion, independent of angiotensin II [73]. Complement-C1q TNF-related protein-1 (CTRP1) was found to be overexpressed in both patients with obesity and hypertension, and to act as an endogenous aldosterone-stimulating factor [74]. Other adipokines such as tumor necrosis factor- $\alpha$ and interleukin- 6 have also been shown to be involved in aldosterone secretion [75]. In vitro and in vivo studies on animal models showed that the adipocyte-derived anorectic hormone leptin could directly promote aldosterone secretion independently of potassium, angiotensin II and adrenocorticotropic hormone levels $[75,76]$. Recent findings suggest that leptin-mediated pathway leading to hypertension development in patients with obesity might be mostly dependent to aldosterone oversecretion in women, and predominantly related to hypothalamic receptor activation and consequent sympathetic nervous system stimulation in men [77]. However, the role of leptin remains unclear. A study did not observe a significant difference in leptin levels among patients with untreated PA compared with healthy individuals [78]. Furthermore, another study showed a postoperative increase in plasma leptin concentration after adrenalectomy in patients with unilateral PA [79]. Interestingly, the role of obesity in the pathogenesis of aldosterone hypersecretion appeared to be relevant in patients with BHA, but not with APA $[80,81]$. These observations suggest that, while aldosterone overproduction is determined by a hormone-secreting tumor in APA patients, obesity might play an important role in aldosterone excess by adipokine-driven pathways in subjects with $\mathrm{BHA}[80,81]$. Vice versa, aldosterone excess might contribute to obesity development through adipose tissue maturation by means of mineralocorticoid receptor activation, thereby leading to a vicious cycle [82].

Higher prevalence of metabolic syndrome and diabetes has been shown in patients with PA compared with essential hypertension [83]. Insulin secretion might be impaired by both aldosterone-induced hypokalemia, and direct aldosterone effects resulting in pancreatic beta-cell dysfunction and apoptosis. Furthermore, hyperaldosteronism-related insulin resistance might be explained by defective expression of glucose transporter 4 , insulin receptor and its related signal transducing factors in skeletal muscle and adipose tissue, as well as increased hepatic gluconeogenesis and endothelial remodeling affecting insulin and glucose peripheral delivery [84]. Finally, recent findings support the coexistence of mild glucocorticoid excess and aldosterone overproduction in PA, suggesting a role for cortisol-driven pathways in the determination of metabolic risk in a proportion of patients with PA [85]. In particular, recent studies showed that cortisol co-secretion in patients with PA might independently contribute to associated metabolic risk 
[86], including a higher risk of impaired glucose metabolism [87] and cardiovascular events [88], compared with patients not displaying concurrent glucocorticoid excess.

\section{Conclusions}

Aldosterone excess, OSA, and obesity could be interconnected within the context of metabolic syndrome through vicious cycle pathogenetic mechanisms. Obesity leads to OSA development through fat deposition within the neck, while OSA could in turn promote obesity. Adipokines released by visceral fat might induce aldosterone overproduction, which could in turn stimulate fat cell differentiation. Moreover, aldosterone excess-induced volume overload contributes to OSA pathogenesis, and intermittent hypoxia might in turn exacerbate RAAS activation. OSA and hyperaldosteronism might both lead to oxidative stress and inflammation, thus favoring the production of oxidized lipidic derivatives which could in turn aggravate aldosterone oversecretion [72]. Furthermore, hyperaldosteronism-related glucose metabolism dysregulation and diabetes [82] might lead to diabetic neuropathy development, which may consequently affect upper airway neural reflexes, thus favoring OSA [64]. Consistent with these evidences, OSA is frequently observed in patients with PA. Conversely, whether PA is more prevalent in patients with OSA compared with the general population remains controversial.

\section{Conflict of Interest}

The authors declare that they have no conflict of interest.

\section{References}

[1] Pimenta E, Calhoun DA, Oparil S. Sleep apnea, aldosterone, and resistant hypertension. Prog Cardiovasc Dis 2009; 51: 371-380

[2] Martínez-García M-A, Navarro-Soriano C, Torres G et al. Beyond resistant hypertension. Hypertension 2018; 72: 618-624

[3] Calhoun DA, Nishizaka MK, Zaman MA et al. Hyperaldosteronism among black and white subjects with resistant hypertension. Hypertension 2002; 40: 892-896

[4] Daugherty SL, Powers JD, Magid D] et al. Incidence and prognosis of resistant hypertension in hypertensive patients. Circulation 2012; 125 : 1635-1642

[5] Calhoun DA, Nishizaka MK, Zaman MA et al. Aldosterone excretion among subjects with resistant hypertension and symptoms of sleep apnea. Chest 2004; 125: 112-117

[6] Di Murro A, Petramala L, Cotesta D et al. Renin-angiotensin-aldosterone system in patients with sleep apnoea: Prevalence of primary aldosteronism. J Renin Angiot Aldoster Syst 2010; 11: 165-172

[7] Funder JW, Carey RM, Mantero F et al. The Management of Primary Aldosteronism: Case Detection, Diagnosis, and Treatment: An Endocrine Society Clinical Practice Guideline. J Clin Endocrinol Metab 2016; 101: 1889-1916

[8] Prejbisz A, Kolodziejczyk-Kruk S, Lenders JWM et al. Primary Aldosteronism and Obstructive Sleep Apnea: Is this a Bidirectional Relationship? Horm Metab Res 2017; 49: 969-976

[9] Mulatero P, Tizzani D, Viola A et al. Prevalence and Characteristics of Familial Hyperaldosteronism. Hypertension 2011; 58: 797-803
[10] Monticone S, D'Ascenzo F, Moretti C et al. Cardiovascular events and target organ damage in primary aldosteronism compared with essential hypertension: A systematic review and meta-analysis. Lancet Diabetes Endocrinol 2018; 6: 41-50

[11] Monticone S, Sconfienza E, D'Ascenzo F et al. Renal damage in primary aldosteronism: A systematic review and meta-analysis. J Hypertens 2020; 38: 3-12

[12] Monticone S, Burrello J, Tizzani D et al. Prevalence and clinical manifestations of primary aldosteronism encountered in primary care practice. J Am Coll Cardiol 2017; 69: 1811-1820

[13] Rossi GP, Bernini G, Caliumi C et al. A prospective study of the prevalence of primary aldosteronism in 1125 hypertensive patients. J Am Coll Cardiol 2006; 48: 2293-2300

[14] Buffolo F, Li Q, Monticone $S$ et al. Primary aldosteronism and obstructive sleep apnea: a cross-sectional multi-ethnic study. Hypertension 2019; 74: 1532-1540

[15] Somers VK, White DP, Amin R et al. Sleep apnea and cardiovascular disease: An American Heart Association/american College of Cardiology Foundation Scientific Statement from the American Heart Association Council for High Blood Pressure Research Professional Education Committee, Council on Clinical Cardiology, Stroke Council, and Council on Cardiovascular Nursing. In collaboration with the National Heart, Lung, and Blood Institute National Center on Sleep Disorders Research (National Institutes of Health). Circulation 2008; 118: $1080-1111$

[16] Marin JM, Agusti A, Villar I et al. Association between treated and untreated obstructive sleep apnea and risk of hypertension. JAMA 2012; 307: 2169-2176

[17] Peppard PE, Young T, Palta M et al. Prospective study of the association between sleep-disordered breathing and hypertension. N Eng J Med 2000; 342: 1378-1384

[18] Williams B, Mancia G, Spiering W et al. 2018 ESC/ESH Guidelines for the management of arterial hypertension. Eur Heart J 2018; 39: 3021-3104

[19] Marin JM, Carrizo S], Vicente E et al. Long-term cardiovascular outcomes in men with obstructive sleep apnoea-hypopnoea with or without treatment with continuous positive airway pressure: an observational study. Lancet 2005; 365: 1046-1053

[20] Redline S, Foody J. Sleep disturbances: time to join the top 10 potentially modifiable cardiovascular risk factors? Circulation 2011; 124: 2049-2051

[21] Gami AS, Olson EJ, Shen WK et al. Obstructive sleep apnea and the risk of sudden cardiac death: A longitudinal study of 10701 adults. J Am Coll Cardiol 2013; 62: 610-616

[22] Senaratna CV, Perret JL, Lodge C] et al. Prevalence of obstructive sleep apnea in the general population: A systematic review. Sleep Med Rev 2017; 34: 70-81

[23] Heinzer R, Vat S, Marques-Vidal P et al. Prevalence of sleep-disordered breathing in the general population: the HypnoLaus study. Lancet Resp Med 2015; 3: 310-318

[24] Drager LF, Togeiro SM, Polotsky VY et al. Obstructive sleep apnea: A cardiometabolic risk in obesity and the metabolic syndrome. J Am Coll Cardiol 2013; 62: 569-576

[25] Ahmad M, Makati D, Akbar S. Review of and updates on hypertension in obstructive sleep apnea. Int J Hypertens. 2017; 1848375:

[26] Ke X, Guo W, Peng $\mathrm{H}$ et al. Association of aldosterone excess and apnea-hypopnea index in patients with resistant hypertension. Sci Rep 2017; 7: 45241

[27] Lloberes P, Lozano L, Sampol G et al. Obstructive sleep apnoea and 24-h blood pressure in patients with resistant hypertension. J Sleep Res 2010; 19: 597-602 
[28] Sim J], Yan EH, Liu IL et al. Positive relationship of sleep apnea to hyperaldosteronism in an ethnically diverse population. J Hypertens 2011; 29: 1553-1559

[29] Florczak E, Prejbisz A, Szwench-Pietrasz E et al. Clinical characteristics of patients with resistant hypertension: the RESIST-POL study. J Hum Hypertens 2013; 27: 678-685

[30] Wolley M], Pimenta E, Calhoun D et al. Treatment of primary aldosteronism is associated with a reduction in the severity of obstructive sleep apnoea. J Hum Hypertens 2017; 31: 561-567

[31] Chen X, Wang R, Zee P et al. Racial/Ethnic Differences in Sleep Disturbances: The Multi-Ethnic Study of Atherosclerosis (MESA). Sleep 2015; 38: 877-888

[32] Ip MS, Lam B, Lauder I] et al. A community study of sleep-disordered breathing in middle-aged Chinese men in Hong Kong. Chest 2001; 119: 62-69

[33] Ip MS, Lam B, Tang LC et al. A community study of sleep-disordered breathing in middle-aged Chinese women in Hong Kong: prevalence and gender differences. Chest 2004; 125: 127-134

[34] Kario K, Chen CH, Park S et al. Consensus Document on Improving Hypertension Management in Asian Patients, Taking Into Account Asian Characteristics. Hypertension 2018; 71: 375-382

[35] Cai A, Zhou Y, Zhang ] et al. Epidemiological characteristics and gender-specific differences of obstructive sleep apnea in a Chinese hypertensive population: A cross-sectional study. BMC Cardiovasc Disord 2017; 17: 8

[36] Ancoli-Israel S, Klauber MR, Stepnowsky C et al. Sleep-disordered breathing in African-American elderly. Am J Resp Crit Care Med 1995; 152: 1946-1949

[37] Redline S, Tishler PV, Hans MG et al. Racial differences in sleep-disordered breathing in African-Americans and Caucasians. Am J Resp Crit Care Med 1997; 155: 186-192

[38] Tu W, Eckert G], Pratt JH et al. Plasma levels of prorenin and renin in blacks and whites: their relative abundance and associations with plasma aldosterone concentration. Am J Hypertens 2012; 25: 1030-1034

[39] Nishizaka MK, Pratt-Ubunama M, Zaman MA et al. Validity of Plasma Aldosterone-to-Renin Activity Ratio in African American and White Subjects With Resistant Hypertension. Am J Hypertens 2005; 18: 805-812

[40] Johnson DA, Thomas S], Abdalla M et al. Association between sleep apnea and blood pressure control among blacks. Circulation 2019; 139: $1275-1284$

[41] Gonzaga C, Bertolami A, Bertolami M et al. Obstructive sleep apnea, hypertension and cardiovascular diseases. J Hum Hypertens 2015; 29: 705-712

[42] Soltysiak M, Miazgowski T, Jaroszyńska E et al. Impaired aldosterone response to the saline infusion test in patients with resistant hypertension and obstructive sleep apnea. Nadcisn Tetn 2015; 19 : 13-18

[43] Yang S-j, Jiang X-T, Zhang X-B et al. Does continuous positive airway pressure reduce aldosterone levels in patients with obstructive sleep apnea? Sleep Breath 2016; 20: 921-928

[44] Moller DS, Lind P, Strunge B et al. Abnormal vasoactive hormones and 24-hour blood pressure in obstructive sleep apnea. Am J Hypertens 2003; 16: 274-280

[45] Zhang J, Tian L, Guo L. Changes of aldosterone levels in patients with type 2 diabetes complicated by moderate to severe obstructive sleep apnea-hypopnea syndrome before and after treatment with continuous positive airway pressure. J Int Med Res 2019; 47: 4723-4733

[46] de Souza F, Muxfeldt ES, Margallo $V$ et al. Effects of continuous positive airway pressure treatment on aldosterone excretion in patients with obstructive sleep apnoea and resistant hypertension: a randomized controlled trial. J Hypertens 2017; 35: 837-844
[47] Casitas R, Martínez-Cerón E, Galera R et al. The effect of treatment for sleep apnoea on determinants of blood pressure control. Eur Resp J 2017; 50: 1701261

[48] Thunström E, Manhem K, Yucel-Lindberg T et al. Neuroendocrine and inflammatory responses to losartan and continuous positive airway pressure in patients with hypertension and obstructive sleep apnea. a randomized controlled trial. Ann Am Thor Soc 2016; 13: 2002-2011

[49] Perger E, Jutant EM, Redolfi S. Targeting volume overload and overnight rostral fluid shift: A new perspective to treat sleep apnea. Sleep Med Rev 2018; 42: 160-170

[50] Kasai T, Bradley TD, Friedman O et al. Effect of intensified diuretic therapy on overnight rostral fluid shift and obstructive sleep apnoea in patients with uncontrolled hypertension. J Hypertens 2014; 32: 673-680

[51] Dudenbostel T, Calhoun DA. Resistant hypertension, obstructive sleep apnoea and aldosterone. J Hum Hypertens 2012; 26: 281-287

[52] Jin ZN, Wei YX. Meta-analysis of effects of obstructive sleep apnea on the renin-angiotensin-aldosterone system. J Geriat Cardiol 2016; 13: $333-343$

[53] Muxfeldt ES, Margallo VS, Guimaraes GM et al. Prevalence and associated factors of obstructive sleep apnea in patients with resistant hypertension. Am J Hypertens 2014; 27: 1069-1078

[54] Pratt-Ubunama MN, Nishizaka MK, Boedefeld RL et al. Plasma aldosterone is related to severity of obstructive sleep apnea in subjects with resistant hypertension. Chest 2007; 131: 453-459

[55] Gonzaga CC, Gaddam KK, Ahmed Ml et al. Severity of obstructive sleep apnea is related to aldosterone status in subjects with resistant hypertension. J Clin Sleep Med 2010; 6: 363-368

[56] Pimenta E, Stowasser M, Gordon RD et al. Increased dietary sodium is related to severity of obstructive sleep apnea in patients with resistant hypertension and hyperaldosteronism. Chest 2013; 143: 978-983

[57] Gaddam K, Pimenta E, Thomas S] et al. Spironolactone reduces severity of obstructive sleep apnoea in patients with resistant hypertension: A preliminary report. J Hum Hypertens 2010; 24: 532-537

[58] Krasinska B, Miazga A, Cofta S et al. Effect of eplerenone on the severity of obstructive sleep apnea and arterial stiffness in patients with resistant arterial hypertension. Pol Arch Med Wewnet 2016; 126: 330-339

[59] Yang L, Zhang H, Cai M et al. Effect of spironolactone on patients with resistant hypertension and obstructive sleep apnea. Clin Exp Hypertens 2016; 38: 464-468

[60] Zhang ZH, Yu Y, Kang YM et al. Aldosterone acts centrally to increase brain renin-angiotensin system activity and oxidative stress in norma rats. American Journal of Physiology. Heart Circul Physiol 2008; 294: H1067-H1074

[61] Monticone S, Viola A, Tizzani D et al. Primary aldosteronism: Who should be screened? Horm Metab Res 2012; 44: 163-169

[62] Ip MS, Lam B, Ng MM et al. Obstructive sleep apnea is independently associated with insulin resistance. Am J Resp Crit Care Med 2002; 165: 670-676

[63] Kim NH. Obstructive sleep apnea and abnormal glucose metabolism. Diabetes Metab J 2012; 36: 268-272

[64] Reutrakul S, Mokhlesi B. Obstructive sleep apnea and diabetes: A state of the art review. Chest 2017; 152: 1070-1086

[65] Barcelo A, Pierola ], Esquinas C et al. Relationship between aldosterone and the metabolic syndrome in patients with obstructive sleep apnea hypopnea syndrome: Effect of continuous positive airway pressure treatment. PloS One 2014; 9: e84362

[66] Lamounier-Zepter V, Bornstein SR, Ehrhart-Bornstein M. Mechanisms of obesity-related hypertension. Horm Metab Res 2004; 36: 376-380 
[67] Rocchini AP, Katch VL, Grekin R et al. Role for aldosterone in blood pressure regulation of obese adolescents. Am J Cardiol 1986; 57 : 613-618

[68] Engeli S, Bohnke J, Gorzelniak K et al. Weight loss and the renin-angiotensin-aldosterone system. Hypertension 2005; 45: 356-362

[69] Dudenbostel T, Ghazi L, Liu M et al. Body mass index predicts 24-hour urinary aldosterone levels in patients with resistant hypertension Hypertension 2016; 68: 995-1003

[70] Li L, Hou X, Geng X et al. Body mass index predicts aldosterone production in hypertensive postmenopausal women. Clin Exp Hypertens 2019; 1-6

[71] Massiera F, Bloch-Faure M, Ceiler D et al. Adipose angiotensinogen is involved in adipose tissue growth and blood pressure regulation. FASEB J 2001; 15: 2727-2729

[72] Goodfriend TL. Obesity, sleep apnea, aldosterone, and hypertension. Curr Hypertens Rep 2008; 10: 222-226

[73] Ehrhart-Bornstein M, Lamounier-Zepter V, Schraven A et al. Human adipocytes secrete mineralocorticoid-releasing factors. Proc Natl Acad Sci USA 2003; 100: 14211-14216

[74] Jeon JH, Kim K-y, Kim JH et al. A novel adipokine CTRP1 stimulates aldosterone production. FASEB J 2008; 22: 1502-1511

[75] Xie D, Bollag WB. Obesity, hypertension and aldosterone: Is leptin the link? J Endocrinol 2016; 230: F7-F11

[76] Huby AC, Antonova G, Groenendyk J et al. Adipocyte-derived hormone leptin is a direct regulator of aldosterone secretion, which promotes endothelial dysfunction and cardiac fibrosis. Circulation 2015; 132: 2134-2145

[77] Faulkner JL, Belin de Chantemele EJ. Sex differences in mechanisms of hypertension associated with obesity. Hypertension 2018; 71: 15-21

[78] Haluzik M, Sindelka G, Widimsky J Jr. et al. Serum leptin levels in patients with primary hyperaldosteronism before and after treatment: Relationships to insulin sensitivity. J Hum Hypertens 2002; 16: 41-45
[79] Torpy DJ, Bornstein SR, Taylor W et al. Leptin levels are suppressed in primary aldosteronism. Horm Metab Res 1999; 31: 533-536

[80] Ohno $\mathrm{Y}$, Sone $\mathrm{M}$, Inagaki N et al. Obesity as a key factor underlying idiopathic hyperaldosteronism. J Clin Endocrinol Metab 2018; 103: 4456-4464

[81] Shibayama Y, Wada N. Relationship Between Visceral Fat and Plasma Aldosterone Concentration in Patients With Primary Aldosteronism. J Endocr Soc 2018; 2: 1236-1245

[82] Kalil GZ, Haynes WG. Sympathetic nervous system in obesity-related hypertension: Mechanisms and clinical implications. Hypertens Res 2012; 35: 4-16

[83] Fallo F, Veglio F, Bertello C et al. Prevalence and characteristics of the metabolic syndrome in primary aldosteronism. J Clin Endocrinol Metab 2006; 91: 454-459

[84] Bothou C, Beuschlein F, Spyroglou A. Links between aldosterone excess and metabolic complications: A comprehensive review. Diabetes Metab 2020; 46: 1-7

[85] Beuschlein F, Reincke M, Arlt W. The impact of Connshing's syndrome - mild cortisol excess in primary aldosteronism drives diabetes risk. J Hypertens 2017; 35: 2548

[86] Arlt W, Lang K, Sitch AJ et al. Steroid metabolome analysis reveals prevalent glucocorticoid excess in primary aldosteronism. J Clin Invest Insight 2017; 2: e93136

[87] Gerards J, Heinrich DA, Adolf $C$ et al. Impaired glucose metabolism in primary aldosteronism is associated with cortisol cosecretion. J Clin Endocrinol Metab 2019; 104: 3192-3202

[88] Nakajima Y, Yamada M, Taguchi R et al Cardiovascular complications of patients with aldosteronism associated with autonomous cortisol secretion. J Clin Endocrinol Metab 2011; 96: 2512-2518 